\title{
Introducing a highly dispersed reduced graphene oxide nano- biohybrid employing chitosan/hydroxyethyl cellulose for controlled drug delivery
}

\author{
Hanieh Mianehrow ${ }^{1}$, Ronak Afshari ${ }^{2}$, Saeedeh Mazinani $^{3 *}$, Farhad Sharif ${ }^{1}$, Majid \\ Abdouss $^{2}$ \\ ${ }^{1}$ Department of Polymer Engineering and Colour Technology, Amirkabir University of Technology, \\ 15875-4413, Tehran, Iran \\ ${ }^{2}$ Department of Chemistry, Amirkabir University of Technology, \\ 15875-4413, Tehran, Iran \\ 3*Amirkabir Nanotechnology Research Institute (ANTRI), Amirkabir University of Technology, \\ 15875-4413,Tehran, Iran \\ a mianehro@aut.ac.ir, ${ }^{\mathrm{b}}$ ronak_afshari@yahoo.com, ${ }^{\mathrm{c}}$ s.mazinani@aut.ac.ir \\ dsharif@aut.ac.ir, ${ }^{\mathrm{d}}$ phdabdouss44@aut.ac.ir
}

*Corresponding Author: Saeedeh Mazinani; E-mail: s.mazinani@aut.ac.ir

Amirkabir Nanotechnology Research Institute (ANTRI), Amirkabir University of Technology, 424 Hafez Ave., Amirkabir University of Technology (Polytechnic of Tehran), Tehran, IRAN. P.O.: 15875-4413. Tel \& Fax No.: (+98) 21-66402441 


\begin{abstract}
In this research, an attempt was made to stabilize reduced graphene oxide ( $\mathrm{rGO}$ ) in all $\mathrm{pH}$ ranges, incorporating both chitosan (CS) and hydroxyethyl cellulose (HEC) to make a proper drug carrier with suitable stability and drug release behavior. The stability of rGO-CS-HEC nanohybrid was assessed using field emission scanning electron microscopy (FE-SEM), ultraviolet-visible spectroscopy (UV-VIS) and Zeta potential measurements. Results depicted that the novel synthesized nanohybrid was stable in all $\mathrm{pH}$ ranges, due to the utilization of HEC, while without incorporation of this material, the rGO-CS nanohybrid aggregated at neutral and alkaline media, due to the ionic nature of chitosan. In addition, drug loading and release behaviour of folic acid (FA), as a model drug, was investigated to assess the role of chitosan on the release behavior of FA from the rGO-CS-HEC nanohybrid in comparison with rGO-HEC and rGO-CS nanohybrids. It was proved that the resultant nanohybrid could release nearly $27 \%$ more FA than the rGO-HEC nanohybrid and only 9\% lower than the rGO-CS nanohybrid during 120 hours. Moreover, the biocompatibility of the resultant nanohybrid was also checked to introduce the novel rGO-CSHEC nanohybrid as a suitable candidate for drug delivery application.
\end{abstract}

Keywords: Nano-biohybrid, Reduced graphene oxide, Chitosan, Hydroxyethyl cellulose, Stabilization, Drug delivery application

\title{
Chemical compounds studied in this article:
}

Chitosan (PubChem CID: 71853); Hydroxyethyl Cellulose (PubChem CID: 24846132); Graphene (PubChem CID: 5462310); Folic Acid (PubChem CID: 6037) 


\section{Introduction}

Graphene oxide with its two-dimensional structure has shown potential applications in the field of targeted drug delivery (Mo et al., 2015; Shi et al., 2013; Wang et al., 2013; Wei et al., 2016; Xie et al., 2016; Yang et al., 2016; Zheng et al., 2015). This nanomaterial with its planar structure is capable of immobilizing different drug molecules, hydrophilic and hydrophobic, by non-covalent bonding or $\pi-\pi$ interaction with high loading capacity, and it can respond to different stimuli for drug release (Hu et al., 2012; Rana et al., 2011; Yang et al., 2008; Zhang et al., 2011). However, the aggregation of GO in electrolyte solutions, such as blood media, restricts its effectiveness for drug delivery application (Hong et al., 2011; Wang and Hu, 2013). Therefore, stabilization of GO suspension in electrolyte media is the most important challenge for any biological application of this injectable drug carrier.

In this regard, functionalization of GO with different water soluble and biocompatible polymers has been practiced to stabilize GO in electrolyte solutions for various biological applications(Bao et al., 2011; Hu et al., 2012; Lee et al., 2011; Liu et al., 2008; Rana et al., 2011). For instance, chitosan (CS) as an ionic biopolymer, has been widely used for stabilization of GO in acidic media to form an effective $\mathrm{pH}$-responsive drug carrier for cancer therapy (Ardeshirzadeh et al., 2015; Duran et al., 2015; Justin and Chen, 2014; Park et al., 2010). However, in contrary to high release rate and $\mathrm{pH}$-responsivity of GO-CS nanohybrid, it starts to aggregate in neutral and alkaline solutions (Fang et al., 2010; Liu et al., 2012), such as blood media and normal cells, due to the deprotonation of chitosan's amino groups (Afshari et al., 2015). This aggregation severely affects the utility of the nanohybrid as a drug carrier, because the nanohybrid must be stable when it passes through the blood media to show the maximum effectiveness and evade the reticuloendothelial system (RES). Therefore, the stabilization of GO-CS nanohybrid in all $\mathrm{pH}$ ranges is crucial for its application as a drug carrier for cancer therapy, although in many researches, less attention has been paid to this important point, and the stability of the nanohybrid has been assessed only in acidic media, unlike the importance of colloidal stability in all $\mathrm{pH}$ ranges for drug delivery systems.

In our previous study (Mianehrow et al., 2015), hydroxyethyl cellulose (HEC), a water soluble, non-ionic derivative of cellulose, was utilized as GO stabilizer for drug delivery application and the resultant nanohybrid presented high and long term stability in different electrolyte media, independent of the ionic nature of the solution. However, there was a conflict between stabilizing 
effect of HEC and its drug release capability. The more HEC content in GO-HEC nanohybrid led to the longer stability of the nanohybrid, and less drug release because of the high interaction between HEC and folic acid (FA) drug, which dominates the drug release efficiency of such nanohybrid and makes the resultant nanohybrid undesirable for drug delivery application.

In the present study, stabilization of GO by incorporation of CS and HEC is assessed for the first time to exploit the advantages of both materials at the same time (Fig.1). HEC is used for stabilization of GO in all $\mathrm{pH}$ ranges and $\mathrm{CS}$ is utilized to get more drug release efficiency. Then, the dual functionalized GO is reduced to enhance its drug loading capacity through $\pi-\pi$ interaction with drug molecules and at the end, loading and release behaviour of folic acid, as a model drug, from rGO-CS-HEC nanohybrid is investigated to check the effect of chitosan on the release rate of the drug from the novel nanocarrier, especially in comparison with rGO-HEC nanohybrid.

\section{Experimental}

\subsection{Materials}

Graphite flake with average lateral size of 20-100 micron, potassium permanganate, nitric acid $67 \%$, sulfuric acid $98 \%$, hydrochloridric acid 37\%, hydrogen peroxide $30 \%$ were purchased from Merck chemicals Co. Folic acid with molecular weight of $441.39 \mathrm{~g} / \mathrm{mol}$, L-ascorbic acid with molecular weight of $176.12 \mathrm{~g} / \mathrm{mol}$ and low molecular weight chitosan (50,000-190,000 Da) were purchased from Sigma Aldrich Co. Hydroxyethyl cellulose with molecular weight of 300,000 g/mol and 1.5 (MS) was purchased from Kelong Chemical Co. (China), and all materials were used as received.

\subsection{Synthesis of GO}

GO was synthesized from natural graphite via preoxidation/thermal expansion of graphite followed by modified Hummer's method (Hummers Jr and Offeman, 1958; McAllister et al., 2007), following the same procedure as our previous work (Mianehrow et al., 2015). At first step, $10 \mathrm{~g}$ of natural graphite flake were mixed with $3: 1$ volume ratio of $\mathrm{H}_{2} \mathrm{SO}_{4}: \mathrm{HNO}_{3}$ for $24 \mathrm{~h}$ at room temperature. Then $400 \mathrm{ml}$ of water was added to the resultant solution for quenching the reaction. The resultant product was then filtered and washed out with water and dried in vacuum. In the 
second step, $2 \mathrm{~g}$ of the product was mixed with $200 \mathrm{ml}$ of $\mathrm{H}_{2} \mathrm{SO}_{4}$ and $6 \mathrm{~g}$ potassium permanganate for $24 \mathrm{~h}$ resulting in a thick paste. Then, $600 \mathrm{ml}$ of distilled water was added to the paste, and the reaction was terminated by addition of aqueous solution of $\mathrm{H}_{2} \mathrm{O}_{2}(30 \mathrm{wt}$. \%), resulting in a yellow brown mixture. Then the mixture was centrifuged and washed out three times with $10 \% \mathrm{HCl}$ solution and then three times with distilled water. The procedure was followed by sonication for 30 minutes to produce GO stable aqueous dispersion. At the end of this process, the mixture was centrifuged for $15 \mathrm{~min}$ at $4500 \mathrm{rpm}$ in order to remove un-exfoliated graphite oxide particles. Synthesized GO full characterization could be found in the supporting information.

\subsection{Preparation of rGO-CS-HEC, rGO-CS and rGO-HEC nanohybrids}

Briefly, $1 \mathrm{ml}$ of as-prepared GO dispersion $(5.4 \mathrm{mg} / \mathrm{ml})$ was added to $20 \mathrm{ml}$ of acetic acid $(0.2 \mathrm{M})$. It was first mixed with CS powder following by 15 minutes sonication, and then mixed with HEC powder by another 15 minutes sonication to produce a crystal clear solution of GO:CS:HEC with optimized mass ratio of 1:3:3. It is necessary to mention that we were aware that at least 1:6 mass ratio of rGO to HEC is required to get a stable suspension of rGO-HEC nanohybrid based on our previous work (Mianehrow et al., 2015) . Therefore, here we kept the ratio of rGO-polymer constant and changed the CS/HEC ratio. However, when the ratio of CS was more than HEC, spontaneous aggregation of the nanohybrid happened, which shows the dominating effect of CS over HEC in those ratios. In addition, in the case of higher HEC ratios, less drug release was expected. Therefore mass ratio of 1:3:3 was obtained as an optimum ratio. Then $25 \mathrm{mg}$ of LAscorbic acid was added to the resultant nanohybrid and the solutions were kept at $70{ }^{\circ} \mathrm{C}$ for 1 hour for partial reduction of the functionalized GO. In the next step, the reduced nanohybrid was dialyzed against deionized water for 3 days for removal of L-ascorbic acid from the suspension. The same route was applied for preparation of $\mathrm{rGO}-\mathrm{CS}$ and $\mathrm{rGO}-\mathrm{HEC}$ nanohybrids with mass ratio of 1:6 (to have the same rGO: polymer ratio) for stability and drug release comparison.

\subsection{FA loaded nanohybrid and in-vitro release analysis}

Loading of FA was performed by mixing of $10 \mathrm{ml}$ of FA (with $80 \mathrm{ppm}$ concentration at $\mathrm{pH}$ of 9) with $15 \mathrm{mg}$ of as-prepared nanohybrid overnight in a dark room. The mixture was then centrifuged 
at $6000 \mathrm{rpm}$ for $1 \mathrm{hr}$ to remove the unloaded drug from the mixture. The drug loading efficiency of the nanocarrier was measured by UV-VIS spectrophotometer and was calculated as follows:

Drug loading efficiency $(\%)=100 \times[$ Total concentration of FA - Concentration of FA in the supernatant) / Total concentration of FA]

The release behaviour of the loaded nanohybrid was monitored in phosphate buffer solution (PBS) with adjusted $\mathrm{pH}$ at 5.3. Briefly, $15 \mathrm{mg}$ of the nanohybrid including $5 \mathrm{ml}$ of the buffer was sealed in a dialysis tube with $12 \mathrm{kDa}$ pore size. The dialysis tube was then immersed in $25 \mathrm{ml}$ of the same saline buffer and placed in a shaking bed with a rotational speed of $150 \mathrm{rpm} .3 \mathrm{ml}$ of release media was withdrawn every hour for the first $10 \mathrm{~h}$ and thereafter every $24 \mathrm{~h}$ until $120 \mathrm{~h}$. Released amount of folic acid in the samples was measured by UV-VIS spectrometer and each measurement has been done for three times.

\subsection{Cytotoxicity of the synthesized nanohybrid}

In vitro cytotoxicity of rGO-CS-HEC nanohybrid at different concentrations of $10,20,50 \mu \mathrm{g} / \mathrm{ml}$ was evaluated by MTT assay. Briefly, human fibroblast (HDF) cells (obtained from National Cell Bank of Iran, Pasteur Institute of Iran, Tehran, Iran) were seeded into 96-well plates (10,000 cells/well) using $100 \mu \mathrm{L}$ RPMI medium and incubated in a humidified $5 \% \mathrm{CO}_{2}$ balanced air incubator at $37^{\circ} \mathrm{C}$ for $24 \mathrm{~h}$. Then, except from control wells, the medium in remaining wells was replaced with $100 \mu \mathrm{L}$ of culture medium containing rGO-CS-HEC nanohybrid with different concentrations, respectively. After incubated at $37^{\circ} \mathrm{C}$ for $24 \mathrm{~h}$, the medium in each well was replaced with $100 \mu \mathrm{L}$ of MTT solution with $0.5 \mathrm{mg} / \mathrm{ml}$ concentration. After continuous incubation for $4 \mathrm{~h}$, the optical density (OD) of each sample was measured at wavelength of 545nm using an Elisa plate reader (STAT FAX 2100, USA). Triplicate samples were analyzed for each experiment.

\subsection{Characterization}

The chemical structure of the nanohybrid was assessed by Bomem Fourier transform infrared (FTIR) spectrometer and its morphology was studied using Hittachi S4160 field emission scanning electron microscope (FE-SEM). X-ray diffraction (XRD) analysis has been done by uning an Inel 
Equinox 3000 diffractometer to study the interlayer spacing of rGO in the nanohybrid. For stability assessments, Analytic Jena (Specord 210) UV-VIS spectrophotometer and ZetaPlus Zeta potential analyzer; Brookhaven Instruments were employed. Fluorescence spectrophotometer, Varian Inc. was utilized for investigation of the molecular interaction between folic acid and the nanohybrid. The size of the nanohybrid particles were measured by particle size analyzer, VASCO-2, Cordouan Technologies, France.

\section{Results and discussion}

\subsection{Characterization of rGO-CS-HEC nanohybrid}

The FT-IR spectra of GO, CS, HEC and rGO-CS-HEC are presented in Fig. 2a. As-prepared GO shows characteristic FTIR peaks at $3438 \mathrm{~cm}^{-1}, 1716 \mathrm{~cm}^{-1}, 1618 \mathrm{~cm}^{-1}, 1180 \mathrm{~cm}^{-1}$ and $1049 \mathrm{~cm}^{-1}$ which are typical indication of vibration of $\mathrm{O}-\mathrm{H}, \mathrm{C}=\mathrm{O}$ groups, $\mathrm{C}=\mathrm{C}$ bonds, $\mathrm{C}-\mathrm{O}-\mathrm{C}$ and $\mathrm{C}-\mathrm{O}$ groups. The FT-IR spectra of CS shows a broad -OH stretch absorption band at $3469 \mathrm{~cm}^{-1}$ and the aliphatic C-H stretch between at $2877 \mathrm{~cm}^{-1}$. As the O-H stretch band and $\mathrm{N}-\mathrm{H}$ stretch band are aligned, it appears as a broad band. Other major absorption bands between 1243 and $1097 \mathrm{~cm}^{-}$ ${ }^{1}$ represent the free primary amino group $\left(-\mathrm{NH}_{2}\right)$ at the $\mathrm{C} 2$ position. The peak at $1656 \mathrm{~cm}^{-1}$ corresponds to the acetylated amino group of chitin, which indicates that the sample was not fully deacetylated. The peak at $1382 \mathrm{~cm}^{-1}$ identifies the $\mathrm{C}-\mathrm{O}$ stretch of primary alcoholic group $\left(-\mathrm{CH}_{2}-\right.$ $\mathrm{OH})$. In FT-IR spectrum of HEC,the bands due to ether C-O-C stretch at $1149-1051 \mathrm{~cm}^{-1}$ appear as a strong peak. The peak at $1149 \mathrm{~cm}^{-1}$ corresponds to the secondary alcohol groups of HEC. In the rGO-CS-HEC spectrum, characteristic peaks of GO, CS and HEC are presented, which confirms the presence of two biopolymers on the surface of GO and the small shift of some peaks depicts the electrostatic interactions between the GO and incorporated bipolymers.

XRD patterns of GO, HEC, CS and the rGO-CS-HEC nanohybrid are investigated as well. As it is depicted in Fig. $2 \mathrm{~b}$, HEC shows a sharp peak at $2 \theta=20.8^{\circ}$ which is characteristic peak of its cellulosic backbone (Chen et al., 2010) and a slight shoulder at $2 \theta=40^{\circ}$ which shows the presence of microcrystalline parts in its structure. Chitosan also shows a sharp peak at $2 \theta=19.4^{\circ}$ related to its backbone and two small peaks at $2 \theta=10^{\circ}$ and $14^{\circ}$ and a broad shoulder around $2 \theta=40^{\circ}$ which 
shows the presence of different crystalline types in the structure of chitosan. As-prepared GO also shows a sharp peak at $2 \theta=11^{\circ}$ which is correspond to the presence of oxygen-containing functional groups between GO layers. It is expected that after the reduction of functional groups of GO, the interlayer distance between GO sheets become significantly shorter, corresponding to a sharp peak at higher degrees. However, rGO-CS-HEC nanohybrid only shows the characteristics peaks of chitosan and HEC and no sign of graphene stacking is observed, which confirms the role of these biopolymers on stabilization of rGO. Moreover, the shoulder observed in case of HEC and CS is less pronounced in the pattern of the resultant nanohybrid, which could be due to the disruption of microcrystalline parts of HEC and CS, suggesting stretching of their chains in the presence of GO.

FE-SEM images of GO and rGO-CS-HEC were used to study the structure and morphology of the synthesized nanohybrid and provide more details about the surface of the nanohybrid (Fig.2c). The SEM image of GO clearly deduce the ultrathin and homogeneous layered structure. After incorporation of CS and HEC to the surface of GO, SEM image shows that the structure of the GO were constantly remained. Also surface changes of GO was clearly observed which confirms the great affinity and interactions between GO surface and two mentioned biopolymers.

The DLS size measurement employing Pade Laplace method showed that the resultant nanohybrid had a particle size range from $33.85 \pm 1.64$ up to $478.57 \pm 22.50 \mathrm{~nm}(\mathrm{n}=3)$. It worth noting that about $97 \%$ of particles mostly included an average size of $478.57 \pm 23.50 \mathrm{~nm}$ and the rest had an average size of $33.85 \pm 1.44 \mathrm{~nm}$.

\subsection{Stability assessments}

Stability assessment of rGO-CS and rGO-CS-HEC in acidic, neutral and alkaline solutions is presented in Fig.3a. As expected, rGO-CS nanohybrid shows stability only in acidic media and start to aggregate in neutral and alkaline solutions, because of the deprotonation of chitosan. In contrast, rGO-CS-HEC nanohybrid seems completely stable in all $\mathrm{pH}$ ranges, which confirms that the steric stabilization strength of non-ionic HEC is able to stabilize rGO-CS nanohybrid independent of the acidity of the solution. 
UV-VIS absorption spectra of rGO-CS-HEC suspension at different concentrations in alkaline media also correlation between the absorbance peaks at $272 \mathrm{~nm}$ and concentrations are presented in Fig.3b. According to the Beer-Lambert law, linear correlation between UV-VIS absorbance and the concentration of a suspension at different concentrations is a proof of its stability (Yang et al., 2010), and as it is depicted in the inset of Fig.3b, rGO-CE-HEC suspension shows the same correlation, which confirms its colloidal stability in alkaline media (the same results were obtained for the nanohybrid in acidic and neutral media (See the supporting information).

Values of zeta potential for rGO-CS and rGO-CS-HEC are presented in Table 1. rGO-CS-HEC nanohybrid shows lower surface charges in comparison to rGO-CS at acidic media, which shows the covering effect of HEC. In addition, by increasing the $\mathrm{pH}$ of the solution, the deprotonation of chitosan is observed. However, the solutions still show high stability, which is directly because of the steric stabilizing strength of HEC, and that is why the stability of such nanohybrid is independent of the $\mathrm{pH}$ of the solution.

Chemical behaviour of rGO-CS and rGO-CS-HEC nanohybrid in different pHs are shown schematically in Fig.4. rGO-CS nanohybrid is stable in acidic media, because of the protonation of chitosan, which causes electrostatic repulsion between functionalized rGO sheets (Fang et al., 2010). By changing the acidity nature of the medium from acidic to neutral and alkaline, chitosan becomes deprotonated, and as a result, disappearance of electrostatic repulsion happens that leads to aggregation of rGO-CS nanohybrid. However, in case of rGO-CS-HEC suspension, the steric hindrance between HEC chains of different nanohybrid sheets, make it stable in all $\mathrm{pH}$ ranges, and at different ionic solutions.

\subsection{Drug loading and release behaviour}

As it is explained in stability assessments part, rGO-CS-HEC nanohybrid is highly stable in all $\mathrm{pH}$ ranges, due to the stabilizing effect of HEC, and this characteristic introduces this nanohybrid as a proper nanocarrier for any biological application. In the next step, for examining the role of chitosan on the drug release behavior of the nanocarrier, FA loading and release behavior is examined. 
The interaction between the nanohybrid and FA drug is assessed by UV-VIS and fluorescence spectroscopy. In Fig.5a, UV-VIS spectra of the supernatant of FA loaded rGO-CS-HEC nanohybrid after centrifugation is compared to the UV-VIS spectra of pure FA solution before loading on the nanohybrid, and it is obvious that a significant amount of FA is loaded by nanohybrid, since its absorbance is noticeably decreased.

Fluorescence emission of FA before and after conjugation with rGO-CS-HEC nanohybrid, with the same concentrations of FA is depicted in Fig. $5 \mathrm{~b}$ to assess the nature of interaction between the samples. The significant quenching of FA emission after conjugation with the nanohybrid, shows the efficient $\pi-\pi$ interaction between FA and rGO-CS-HEC, and proves that even after presence of HEC and CS on the surface of GO, it still has a great surface area for making $\pi-\pi$ interaction with FA, especially because of its partial reduction by L-ascorbic acid. This observation is confirmed by high calculated drug loading capacity of the nanohybrid which is $92 \pm 2 \%$ ( $\mathrm{p}<0.05$, $\mathrm{n}=3$ ), and it depends on hydrogen bonding also $\pi-\pi$ interaction between rGO-CS-HEC and FA.

Fig.6 shows a schematic of drug loading on rGO-CS-HEC nanohybrid. It is well known that CS and HEC chains have the affinity to be conjugated to the carboxyl groups of GO on its edges. Therefore, both materials occupy some places in the edges of GO not its surface, and that is why a huge surface area of the nanohybrid would still be accessible for drug molecules to make $\pi-\pi$ interaction. In addition, hydrogen bonding between FA and adsorbed CS and HEC could be also possible, which explains high drug loading capacity of the nanohybrid.

Release behaviour of FA from rGO-CS-HEC nanohybrid in PBS solution with $\mathrm{pH}$ of 5.3, (which has the same acidity as cancer cells(Du et al., 2011)), is presented in Fig.7a. As it is mentioned in our previous work (Mianehrow et al., 2015), FA release from the GO-HEC nanohybrid is quite low, which is due the low affinity of FA to acidic media and the strong interaction between FA and HEC. In addition, it was observed that by increasing the HEC amount in the nanohybrid, the FA release become significantly less (nearly $3 \%$ release after $72 \mathrm{~h}$ for GO-HEC (1:25)), which shows the dominating role of HEC on release behaviour. This characteristic reduces the effectiveness of the nanohybrid as a drug carrier, even if it shows superb stability in different ionic media. Therefore, chitosan is utilized in this work to improve the release efficiency of the nanohybrid, because of its ionic nature. Results depict that rGO-CS-HEC nanohybrid releases more drug than the control sample of rGO-HEC nanohybrid. As it is observable in Fig 7a, rGO- 
HEC sample releases up to $13 \%$ of FA during $120 \mathrm{hr}$, while rGO-CS-HEC nanohybrid releases $30 \%$ of FA during the same time, which is a significant increase in comparison to our control sample of rGO-HEC. Moreover, it is noticeable that the rGO-CS-HEC sample releases only $9 \%$ less FA than rGO-CS nanohybrid, which confirms that the resultant nanohybrid could show the high stability and more release rate at the same time.

Cytotoxicity of rGO-CS-HEC (with concentrations of 10, 20 and $50 \mu \mathrm{g} / \mathrm{ml}$ ) exposed to human fibroblast (HDF) cells is investigated within $24 \mathrm{~h}$ to assess the biocompatibility of the proposed nanocarrier and to get comparable results according to the cytotoxicity results of pure graphene oxide exposed to the same cell line in the previous published researches (Wang et al., 2011) (Fig.7b). Results prove suitable biocompatibility of the nanohybrid within 24 h. Sample with 10 $\mu \mathrm{g} / \mathrm{ml}$ concentration shows almost no toxicity. Even the sample at $50 \mu \mathrm{g} / \mathrm{ml}$ concentration, shows more than $80 \%$ of cell viability, which confirms the biocompatibility of the rGO-CS-HEC nanohybrid. Consequently, the introduced nanohybrid could be utilized as a proper drug carrier, because of its high stability in all $\mathrm{pH}$ ranges and in different ionic media, controllable drug release and biocompatibility.

\section{Conclusions}

With respect to the importance of the stability of a drug carrier in biological media, an attempt was made to introduce the novel nano-biohybrid based on reduced graphene oxide decorated with chitosan and hydroxylethyl cellulose that is highly stable in all $\mathrm{pH}$ ranges and ionic media. HEC was used as a non-ionic, soluble and biocompatible stabilizer for $\mathrm{rGO}$, and CS was utilized to improve the low drug release characteristic of HEC contained nanohybrid. Results showed that steric hindrance between HEC chains can stabilize rGO in all $\mathrm{pH}$ ranges, and CS is able to enhance the drug release behaviour from the resultant nanocarrier significantly, due to its ionic nature. In addition, the nanohybrid showed high loading capacity, which represents the effect of hydrogen bonding and $\pi-\pi$ interactions between rGO-CS-HEC and FA drug. Therefore, considering the before-mentioned results, it seems that rGO-CS-HEC nanohybrid can be introduced as a promising nanocarrier for drug delivery application, because of its stability, high loading capacity, controllable drug release and biocompatibility. 


\section{Acknowledgement}

The authors would like to express their sincere gratitude to Iran National Science Foundation "INSF" (Grant No. 90004627) for supporting this research.

\section{References:}

Afshari, R., Mazinani, S., Abdouss, M., 2015. Nanohybrid Nanoparticles Based on Chitosan/Functionalized Carbon Nanotubes as Anti-HIV Nanocarrier. NANO 10, 1550010.

Ardeshirzadeh, B., Anaraki, N.A., Irani, M., Rad, L.R., Shamshiri, S., 2015. Controlled release of doxorubicin from electrospun PEO/chitosan/graphene oxide nanocomposite nanofibrous scaffolds. Materials Science and Engineering: $C$ 48, 384-390.

Bao, H., Pan, Y., Ping, Y., Sahoo, N.G., Wu, T., Li, L., Li, J., Gan, L.H., 2011. Chitosan-Functionalized Graphene Oxide as a Nanocarrier for Drug and Gene Delivery. Small 7, 1569-1578.

Chen, R., Yi, C., Wu, H., Guo, S., 2010. Degradation kinetics and molecular structure development of hydroxyethyl cellulose under the solid state mechanochemical treatment. Carbohydrate Polymers 81 , 188-195.

Du, J.-Z., Du, X.-J., Mao, C.-Q., Wang, J., 2011. Tailor-made dual pH-sensitive polymer-doxorubicin nanoparticles for efficient anticancer drug delivery. Journal of the American Chemical Society 133, 1756017563.

Duran, N., Martinez, S.T., P Silveira, C., Durán, M., CM de Moraes, A., B Simoes, M., L Alves, O., J Favaro, W., 2015. Graphene oxide: a carrier for pharmaceuticals and a scaffold for cell interactions. Current topics in medicinal chemistry 15, 309-327.

Fang, M., Long, J., Zhao, W., Wang, L., Chen, G., 2010. pH-responsive chitosan-mediated graphene dispersions. Langmuir 26, 16771-16774.

Hong, B.J., Compton, O.C., An, Z., Eryazici, I., Nguyen, S.T., 2011. Successful stabilization of graphene oxide in electrolyte solutions: enhancement of biofunctionalization and cellular uptake. ACS nano 6, 63-73.

Hu, H., Yu, J., Li, Y., Zhao, J., Dong, H., 2012. Engineering of a novel pluronic F127/graphene nanohybrid for $\mathrm{pH}$ responsive drug delivery. Journal of Biomedical Materials Research Part A 100, 141-148.

Hummers Jr, W.S., Offeman, R.E., 1958. Preparation of graphitic oxide. Journal of the American Chemical Society 80, 1339-1339.

Justin, R., Chen, B., 2014. Characterisation and drug release performance of biodegradable chitosangraphene oxide nanocomposites. Carbohydrate Polymers 103, 70-80.

Lee, D.Y., Khatun, Z., Lee, J.-H., Lee, Y.-k., In, I., 2011. Blood compatible graphene/heparin conjugate through noncovalent chemistry. Biomacromolecules 12, 336-341.

Liu, J., Guo, S., Han, L., Ren, W., Liu, Y., Wang, E., 2012. Multiple pH-responsive graphene composites by non-covalent modification with chitosan. Talanta 101, 151-156.

Liu, Z., Robinson, J.T., Sun, X., Dai, H., 2008. PEGylated nanographene oxide for delivery of water-insoluble cancer drugs. Journal of the American Chemical Society 130, 10876-10877.

McAllister, M.J., Li, J.-L., Adamson, D.H., Schniepp, H.C., Abdala, A.A., Liu, J., Herrera-Alonso, M., Milius, D.L., Car, R., Prud'homme, R.K., 2007. Single sheet functionalized graphene by oxidation and thermal expansion of graphite. Chemistry of Materials 19, 4396-4404. 
Mianehrow, H., Moghadam, M.H.M., Sharif, F., Mazinani, S., 2015. Graphene-oxide stabilization in electrolyte solutions using hydroxyethyl cellulose for drug delivery application. International journal of pharmaceutics 484, 276-282.

Mo, R., Jiang, T., Sun, W., Gu, Z., 2015. ATP-responsive DNA-graphene hybrid nanoaggregates for anticancer drug delivery. Biomaterials 50, 67-74.

Park, J.H., Saravanakumar, G., Kim, K., Kwon, I.C., 2010. Targeted delivery of low molecular drugs using chitosan and its derivatives. Advanced drug delivery reviews 62, 28-41.

Rana, V.K., Choi, M.C., Kong, J.Y., Kim, G.Y., Kim, M.J., Kim, S.H., Mishra, S., Singh, R.P., Ha, C.S., 2011. Synthesis and Drug-Delivery Behavior of Chitosan-Functionalized Graphene Oxide Hybrid Nanosheets. Macromolecular Materials and Engineering 296, 131-140.

Shi, S., Yang, K., Hong, H., Valdovinos, H.F., Nayak, T.R., Zhang, Y., Theuer, C.P., Barnhart, T.E., Liu, Z., Cai, W., 2013. Tumor vasculature targeting and imaging in living mice with reduced graphene oxide. Biomaterials.

Wang, H., Hu, Y.H., 2013. Electrolyte-induced precipitation of graphene oxide in its aqueous solution. Journal of Colloid and Interface Science 391, 21-27.

Wang, K., Ruan, J., Song, H., Zhang, J., Wo, Y., Guo, S., Cui, D., 2011. Biocompatibility of graphene oxide. Nanoscale Res Lett 6, 1-8.

Wang, Y., Huang, R., Liang, G., Zhang, Z., Zhang, P., Yu, S., Kong, J., 2013. MRI-Visualized, Dual-Targeting, Combined Tumor Therapy Using Magnetic Graphene-Based Mesoporous Silica. Small.

Wei, Y., Zhou, F., Zhang, D., Chen, Q., Xing, D., 2016. A graphene oxide based smart drug delivery system for tumor mitochondria-targeting photodynamic therapy. Nanoscale.

Xie, M., Lei, H., Zhang, Y., Xu, Y., Shen, S., Ge, Y., Li, H., Xie, J., 2016. Non-covalent modification of graphene oxide nanocomposites with chitosan/dextran and its application in drug delivery. RSC Advances 6, 93289337.

Yang, H., Bremner, D.H., Tao, L., Li, H., Hu, J., Zhu, L., 2016. Carboxymethyl chitosan-mediated synthesis of hyaluronic acid-targeted graphene oxide for cancer drug delivery. Carbohydrate polymers 135, 72-78.

Yang, Q., Pan, X., Huang, F., Li, K., 2010. Fabrication of high-concentration and stable aqueous suspensions of graphene nanosheets by noncovalent functionalization with lignin and cellulose derivatives. The Journal of Physical Chemistry C 114, 3811-3816.

Yang, X., Zhang, X., Liu, Z., Ma, Y., Huang, Y., Chen, Y., 2008. High-efficiency loading and controlled release of doxorubicin hydrochloride on graphene oxide. The Journal of Physical Chemistry C 112, 17554-17558. Zhang, R., Hummelgård, M., Lv, G., Olin, H., 2011. Real time monitoring of the drug release of rhodamine B on graphene oxide. Carbon 49, 1126-1132.

Zheng, Q., Cai, Z., Ma, Z., Gong, S., 2015. Cellulose nanofibril/reduced graphene oxide/carbon nanotube hybrid aerogels for highly flexible and all-solid-state supercapacitors. ACS applied materials \& interfaces 7, 3263-3271. 

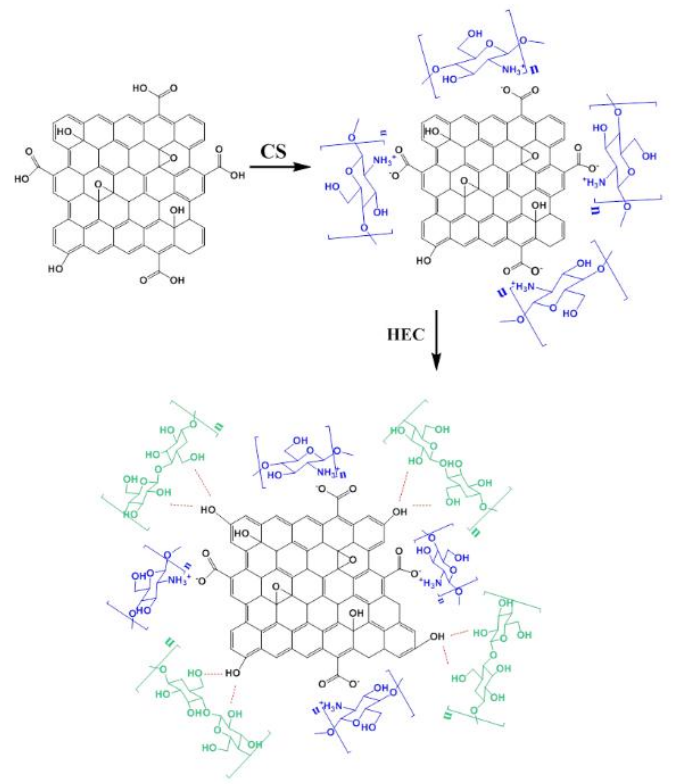


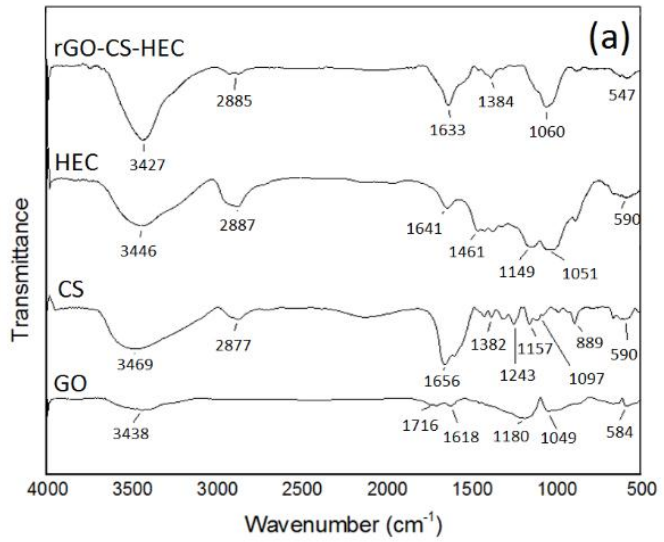

(b)

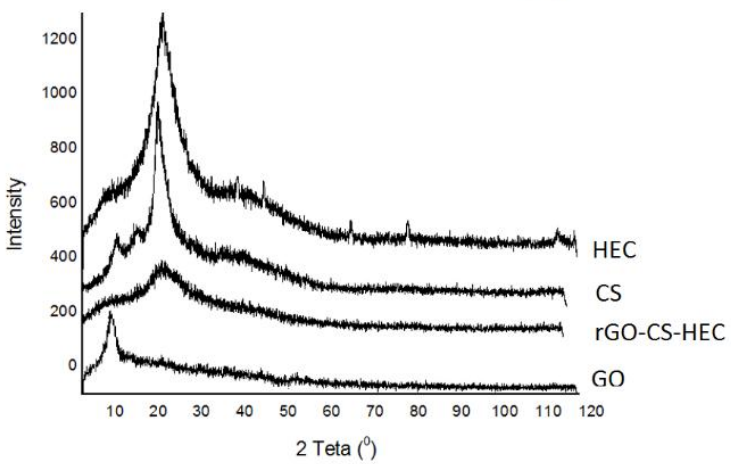

(c)
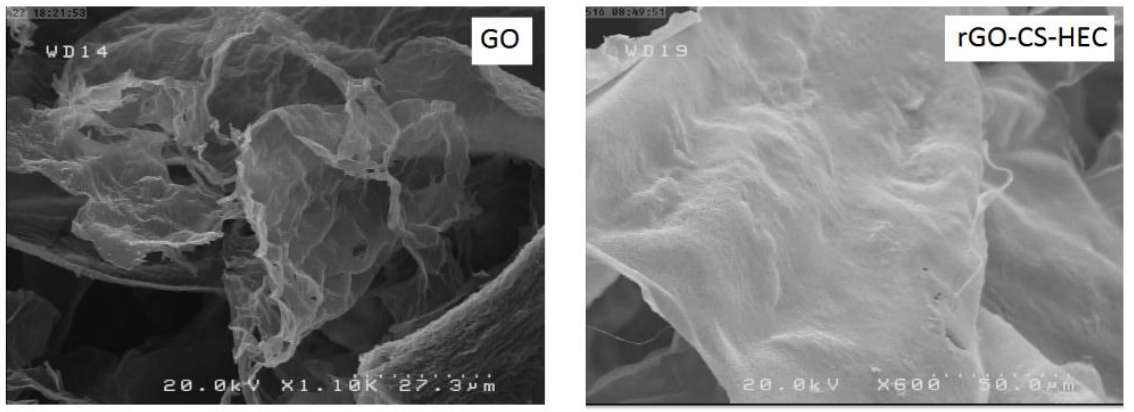


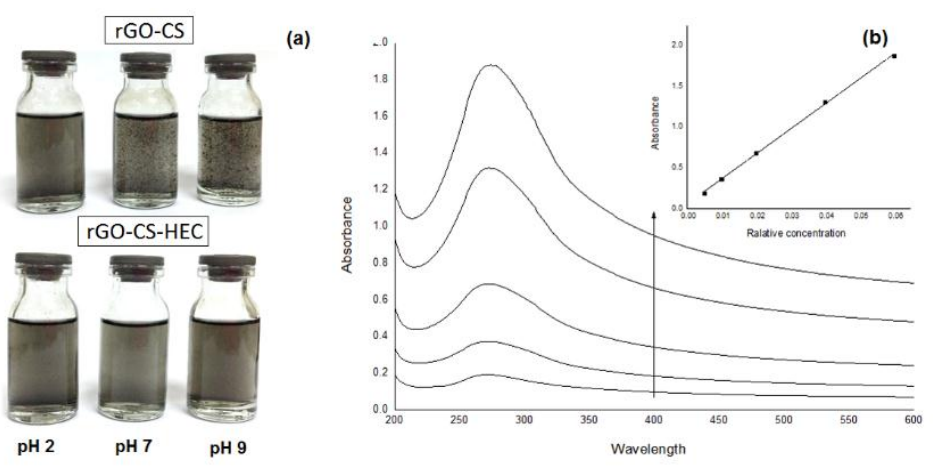




\section{rGO-CS}

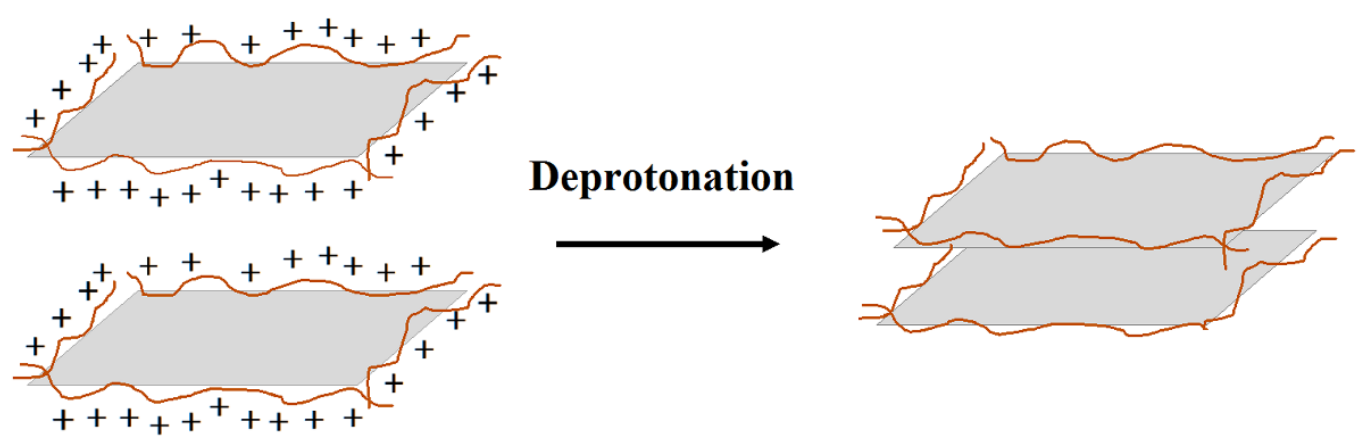

\section{rGO-CS-HEC}
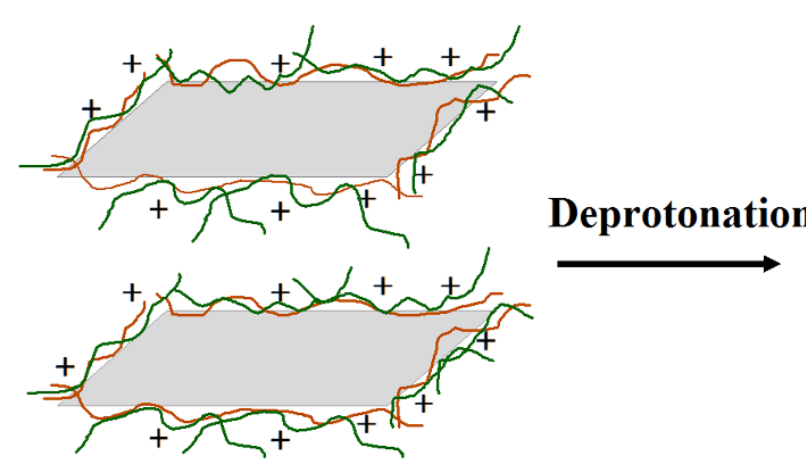

$\mathbf{p H}=\mathbf{2}$

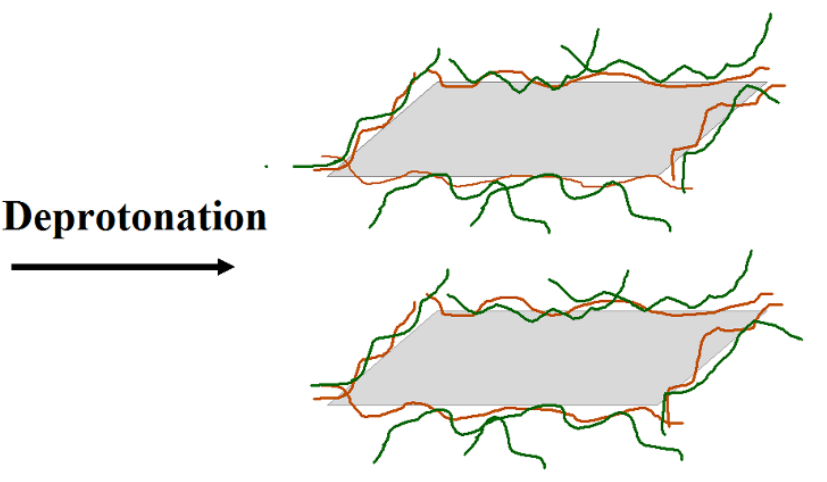

$\mathbf{p H}=7,9$ 

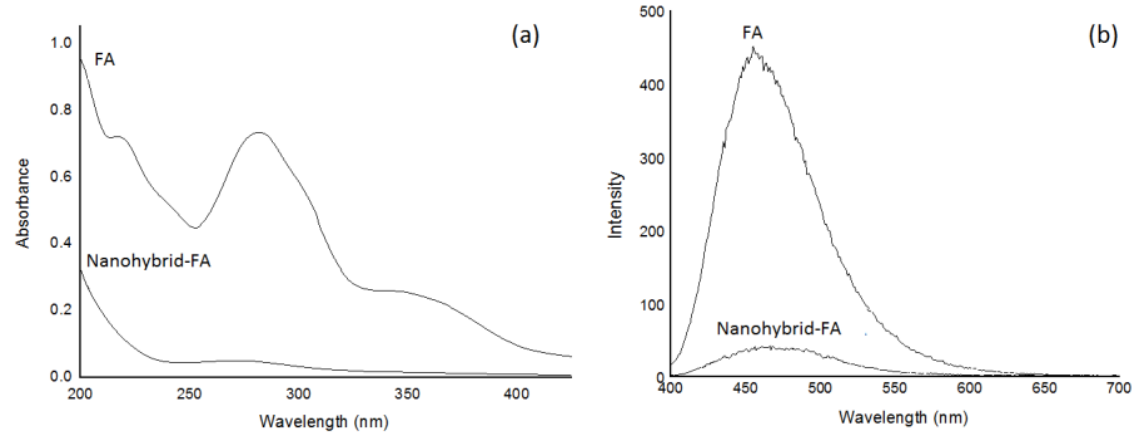


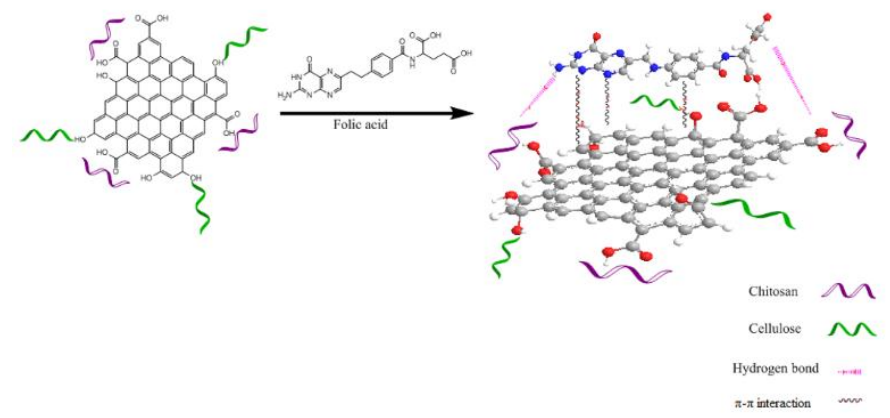



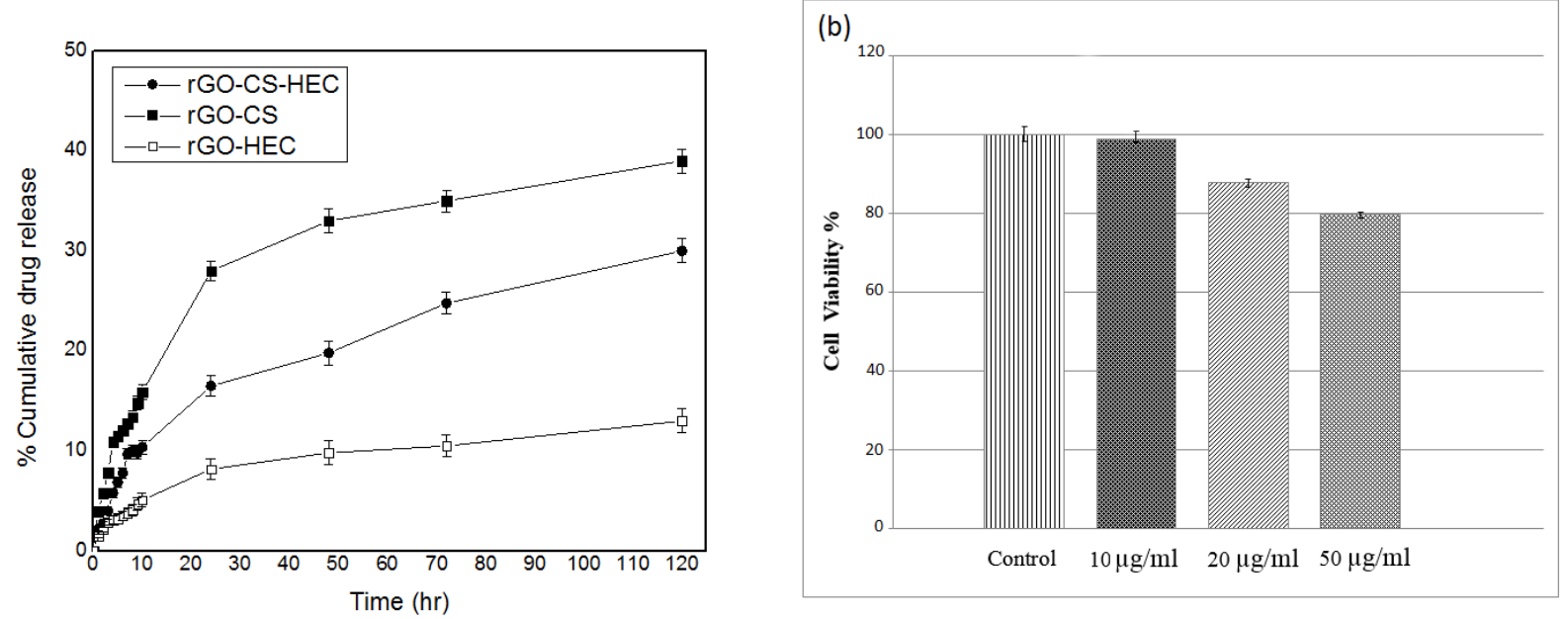


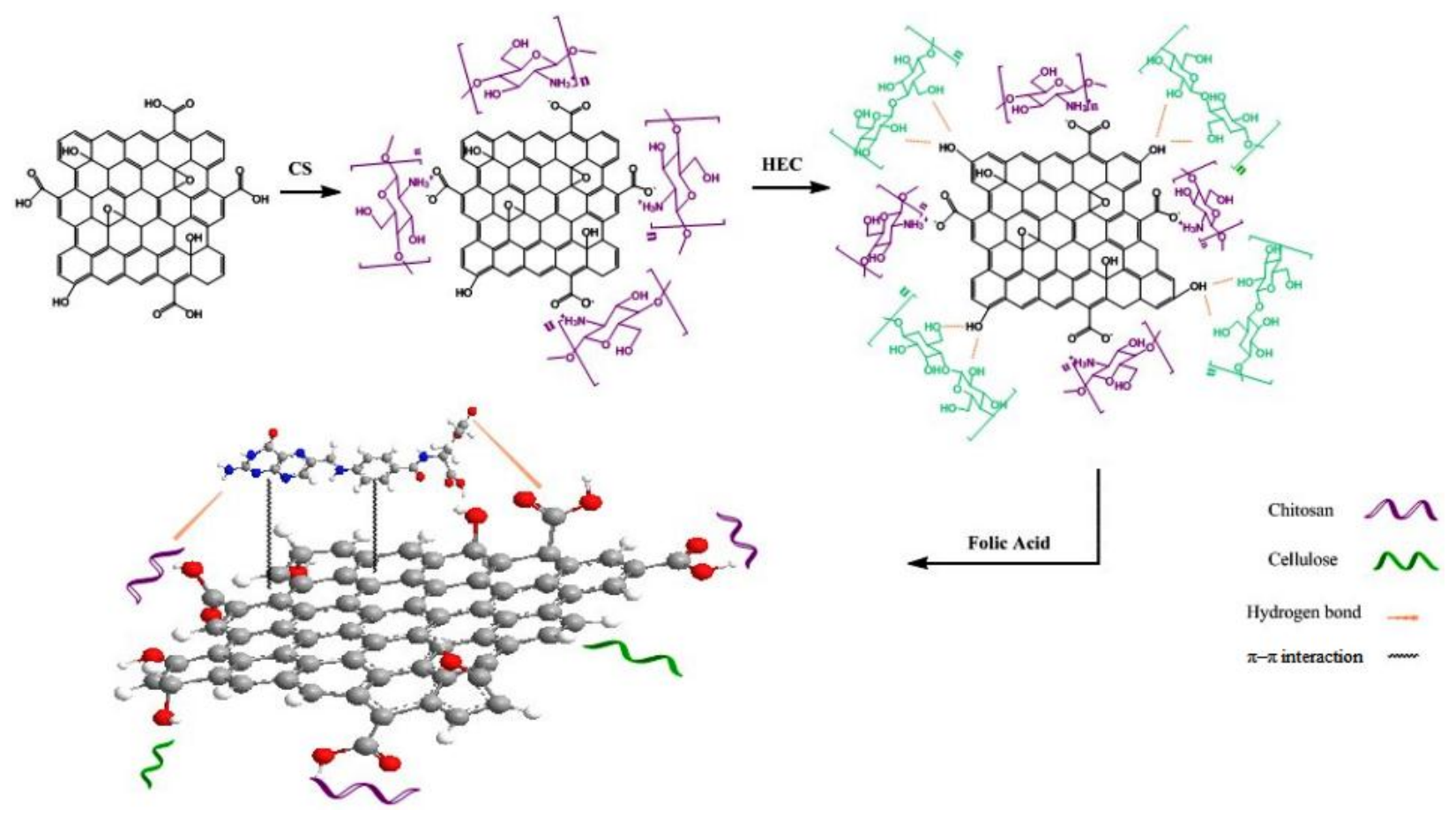




\section{Tables:}

Table 1. Zeta potential values for rGO-CS and rGO-CS-HEC

\begin{tabular}{lccc}
\hline & $\mathrm{pH}$ & $\mathrm{pH}$ & $\mathrm{pH} \mathrm{9}$ \\
\hline rGO-CS-HEC & $+8.5 \mathrm{mV}$ & $+4.3 \mathrm{mV}$ & $-18.2 \mathrm{mV}$ \\
rGO-CS & $+20.7 \mathrm{mV}$ & aggregated & aggregated \\
\hline
\end{tabular}




\section{Figure captions:}

Figure 1. Schematic of rGO-CS-HEC nanohybrid synthesis

Figure 2. FT-IR spectra (a) and XRD patterns (b) of GO, CS, HEC and rGO-CS-HEC nanohybrid, FESEM images of GO and rGO-CS-HEC nanohybrid (c)

Figure 3. Digital images of rGO-CS and rGO-CS-HEC nanohybrid in three different pHs (a), UV-VIS spectra of rGO-CS-HEC suspension at different concentrations in alkaline media (Inset: correlation between the absorbance of the nanohybrid at $272 \mathrm{~nm}$ and its concentration) (b)

Figure 4. Response of rGO-CS and rGO-CS-HEC nanohybrid toward neutral and alkaline media

Figure 5. UV-VIS spectra of supernatant solution of FA loaded nanohybrid after centrifugation and pure FA (a), Fluorescence spectra of FA and FA loaded nanohybrid (excitation wavelength $360 \mathrm{~nm}$ ) (b)

Figure 6. Schematic of folic acid loading on rGO-CS-HEC nanohybrid

Figure 7. Release behavior of FA from rGO-CS-HEC, rGO-CS and rGO-HEC nanohybrids in PBS and $\mathrm{pH}=5.3$ (a), MTT assay of rGO-CS-HEC nanohybrid at different concentrations for 24 hours (b) (The data points are average of three independent experiments. Error bars represent the range over which the values were observed) 